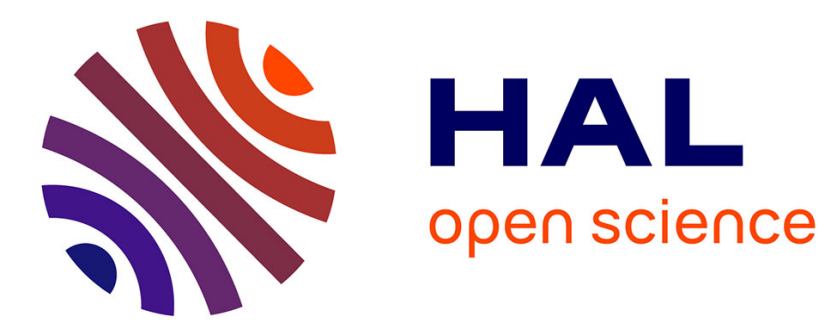

\title{
Fragmentation of magnetic particle aggregates in turbulence
}

\author{
H. M. de La Rosa Zambrano, G. Verhille, P. Le Gal
}

\section{To cite this version:}

H. M. de La Rosa Zambrano, G. Verhille, P. Le Gal. Fragmentation of magnetic particle aggregates in turbulence. Physical Review Fluids, 2018, 3 (8), 10.1103/physrevfluids.3.084605 . hal-01924650

\section{HAL Id: hal-01924650 \\ https://hal.science/hal-01924650}

Submitted on 16 Nov 2018

HAL is a multi-disciplinary open access archive for the deposit and dissemination of scientific research documents, whether they are published or not. The documents may come from teaching and research institutions in France or abroad, or from public or private research centers.
L'archive ouverte pluridisciplinaire HAL, est destinée au dépôt et à la diffusion de documents scientifiques de niveau recherche, publiés ou non, émanant des établissements d'enseignement et de recherche français ou étrangers, des laboratoires publics ou privés. 


\title{
Fragmentation of magnetic particle aggregates in turbulence
}

\author{
H. M. De La Rosa Zambrano, G. Verhille, and P. Le Gal \\ Aix Marseille Université, CNRS, Centrale Marseille, IRPHE, 13013 Marseille, France
}

(Received 6 February 2018; published 24 August 2018)

\begin{abstract}
Particle aggregates are frequently encountered in many natural and industrial environments. We describe here the stationary state of the fragmentation process of inertial scale particle aggregates in turbulence, i.e., when the particles and the aggregates are larger than the Kolmogorov dissipative scale $\eta$. For this purpose, we place at the initial time a large aggregate of millimetric, nearly neutrally buoyant magnetic particles in a high-Reynoldsnumber turbulent von Kármán flow. Turbulent fluctuations impose external stresses that tend to fragment the initial and the subsequent aggregates, contrary to the magnetic dipoles that impose torques and forces on the magnets responsible for cohesion. Using video image analyses, we perform the three-dimensional reconstruction of the aggregates and measure their characteristic sizes. The average number of particles inside each aggregate can then be deduced as a function of the intensity of turbulence. Assuming a Kolmogorov inertial scaling law for the turbulent velocity increments, we predict theoretically an aggregate mean size which is in agreement with our experimental results.
\end{abstract}

DOI: 10.1103/PhysRevFluids.3.084605

\section{INTRODUCTION}

The study of aggregation and fragmentation of flocs in turbulent flows is of great importance in engineering processes such as in the paper or textile making industry [1] where aggregation of particles or fibers is harmfully controlled. Aggregation is also very frequently observed in natural systems where it concerns a huge range of scales from nano- or microorganic particles that can be transported by winds or marine currents [2] to dust grains and even rocks that aggregate in accretion disks during planet formation [3]. If the effects of turbulence on aggregation and fragmentation have been widely studied for particles whose size is within the viscous range of turbulence [4,5], the consequences within the inertial range are poorly documented and not yet understood [6] despite their tremendous importance.

In the present study, we analyze the effect of a turbulent flow acting on aggregates of magnetic beads whose sizes are within the inertial range of turbulence. We performed a three-dimensional video image analysis to detect the aggregates and measure their characteristic sizes. While lowintensity turbulence allows large aggregates, strong turbulence favors small aggregates, an average size being reached when the fragmentation by turbulence is not strong enough to continue the breaking towards smaller scales. Using Kolmogorov scaling arguments, we propose a theoretical model whose predictions are validated by our experiments that are performed in a fully turbulent von Kármán flow. We choose millimetric magnetic particles because the separation force between two adjacent magnets is well characterized and relatively uniform through the thousands of particles we use. Then we will use a fractal description of the aggregate shapes to measure their dimensions in order to predict their average size as a function of the turbulence intensity.

\section{EXPERIMENTAL SETUP AND MEASUREMENT TECHNIQUES}

The turbulence is generated in a transparent acrylic cubic container filled with 81 of water by the counterrotation of two impellers of radius $R=8.6 \mathrm{~cm}$, fitted with straight blades $(h=5 \mathrm{~mm})$. Each 


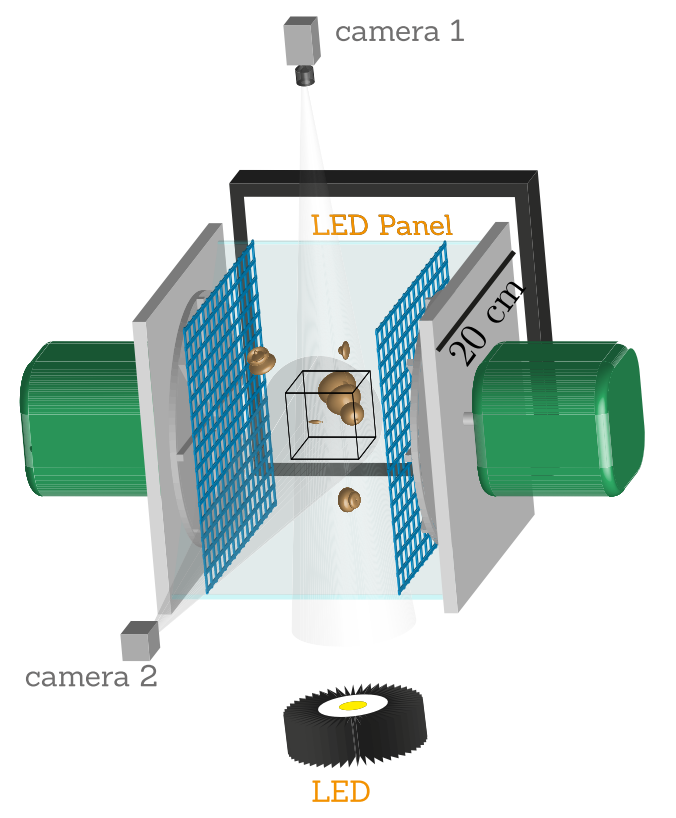

FIG. 1. Sketch of the turbulent von Kármán flow setup.

impeller can rotate independently at a controlled frequency $f$ ranging from 8.5 to $30 \mathrm{~Hz}$. In our study, these two rotation frequencies are equal in order to generate a classical von Kármán flow. Figure 1 shows a sketch of our setup that differs from classical installations [7,8] by the presence of two acrylic grids parallel to the disks. The purpose of these 3-mm mesh grids is to avoid the aggregates to get close to and break on the rotating disks. Visualization is performed through two perpendicular sides of the container. With the help of light-emitting diode (LED) light sources, diffused by a translucent screen, a homogeneous backlight is obtained and used to image the aggregates, allowing good contrast and depth of the visual field.

In order to characterize the turbulence, we have measured its properties by particle image velocimetry $(\mathrm{PIV})[9]$ in an $(11 \times 17)-\mathrm{cm}^{2}$ section of a meridional plane between the grids in the absence of magnetic particles. Since the volume fraction of particles is small enough, we can neglect their backreaction on the flow. Let us note also that at each point $\mathbf{x}$ the turbulent kinetic energy $\left\langle u^{\prime}(\mathbf{x})^{2}\right\rangle$ is at least 5 times the mean flow energy $\langle u(\mathbf{x})\rangle^{2}$. From the two-dimensional PIV velocity fields, we can also measure the energy dissipation rate which is estimated using the longitudinal second-order velocity structure function $D_{L L}(r) \equiv\left\langle[u(\mathbf{x}+r, t)-u(\mathbf{x}, t)]^{2}\right\rangle$, where $r$ is the spatial variable [10-12]. Figure 2(a) shows the variation of $D_{L L}(r)^{3 / 2} / r$ (normalized by the Kolmogorov constant $C_{K}$ ) as a function of $r$ for different disk frequencies. In each case, the inertial regime corresponds to the plateaus extending between $\sim 1 \mathrm{~mm}$ (larger than $\eta$ because of the limited resolution of the PIV) and the integral scale $L_{i} \sim 3 \mathrm{~cm}$. The typical length $L$ of an aggregate is around $1 \mathrm{~cm}$ and thus is smaller than the integral scale of turbulence. At this scale the pressure force induced by the mean flow is much smaller than the one due to turbulence. Hence, the aggregates will be mainly sensitive to the turbulent fluctuations. The energy dissipation rate $\epsilon$ is given by the amplitude of these plateaus using a Kolmogorov constant $C_{K}=2.12$ [13]. The variation of $\epsilon$ with the rotation frequency $f$ is plotted in Fig. 2(b). As expected, $\epsilon$ varies with the third power of $f, \epsilon=(2 \pi \alpha)^{3} R^{2} f^{3}$, in agreement with classical results in turbulent von Kármán flow [7,14], where $\alpha=3.24 \times 10^{-2}$. Table I summarizes the parameters of turbulence.

The 1000 beads used for the experiments were made from 1-mm-diam spherical neodymium magnets. In order to characterize the magnetic interaction, the separation force of a sample of 
(a)

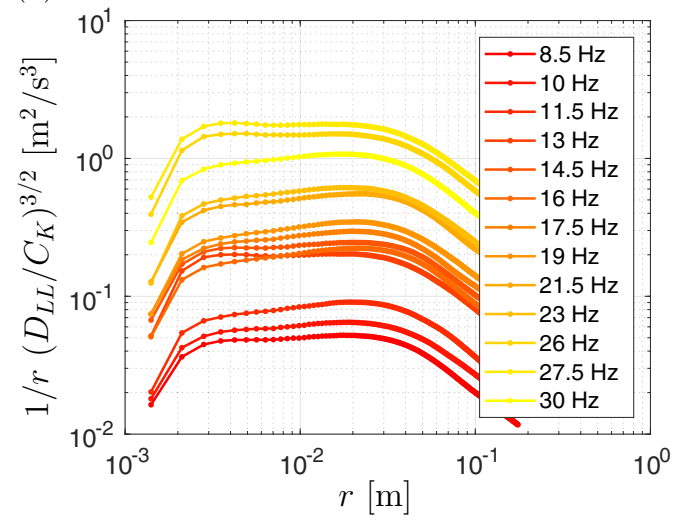

(b)

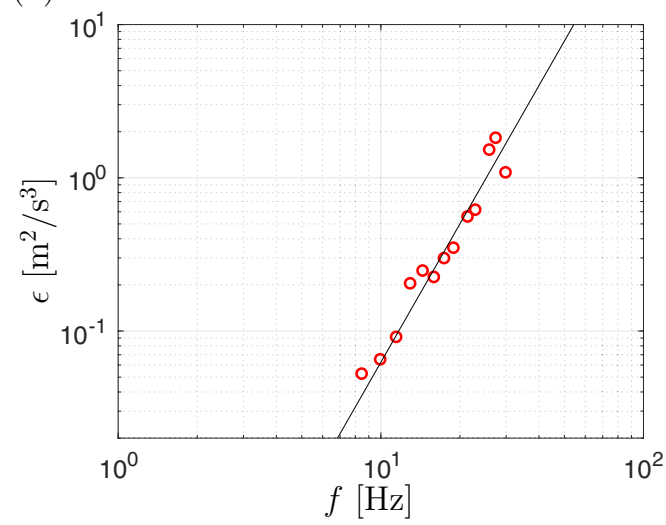

FIG. 2. (a) Normalized longitudinal second-order velocity structure function for the different impeller rotation frequencies $f$. (b) Energy dissipation rate $\epsilon$ as a function of the impellers rotation frequency $f$.

magnetic bead pairs is measured when the pairs are separated by a stepper motor. At contact, the separation force is equal to $0.135 \mathrm{~N}$. Figure 3(a) shows the evolution of the attractive force between two magnets with parallel magnetic moments $\mathbf{m}$. As expected, this force decreases as $r_{b}^{-4}$, where $r_{b}$ is the distance between the two dipoles [15]. We note two important features that prevent the direct use of these magnetic beads: First, since their density is around 7, they would sink in water to the bottom of the container, and second, the separation force at contact would be too large compared to the expected hydrodynamic force, thus preventing any fragmentation of the clusters by turbulence. Because of these two difficulties, we coated the magnets with a layer of wax, which has two benefic effects: First, it reduces the apparent density of the particles and second, by increasing the bead diameter, it decreases the cohesion force at contact. The characteristics of the final wax-coated beads are presented in Figs. 3(b)-3(d), where it can be seen that their shape is ovoidal because of the manufacturing process dipping them into melted wax. They have an aspect ratio around 1.5, an average main axis equal to $3.7 \mathrm{~mm}$, and a mean density of 1.14 . Furthermore, the attractive force $F_{B}$ is decreased and equal to $4 \times 10^{-4} \mathrm{~N}$ for a separation distance of $3.7 \mathrm{~mm}$ corresponding to the average major axis along which the magnetic dipole is aligned. Using the 1000-magnetic-particle volume and the volume of water between the grids, we can evaluate the solid volume fraction of our system to be around 3\%. This low value indicates that the particle laden flow is dilute and that the probability for two particles to meet and merge in the turbulent flow is small. To test the eventual role of buoyancy during the fragmentation process, some of our experimental runs were performed with salty water having a density of 1.14 . As we will see later, very limited effects of sedimentation were detected and most of our experimental runs were finally realized with fresh water.

TABLE I. Principal characteristics of turbulence.

\begin{tabular}{lc}
\hline \hline Parameter & Value \\
\hline Energy dissipation $\epsilon$ & $0.05-2 \mathrm{~m}^{2} / \mathrm{s}^{3}$ \\
Taylor microscale $\lambda=\sqrt{15 \nu u_{\mathrm{rms}}^{2} / \epsilon}$ & $1.6-3.2 \mathrm{~mm}$ \\
Taylor-Reynolds number $\operatorname{Re}_{\lambda}=\sqrt{15 / \nu \epsilon} u_{\mathrm{rms}}^{2}$ & $600-1000$ \\
Kolmogorov length scale $\eta=\left(\nu^{3} / \epsilon\right)^{1 / 4}$ & $28-65 \mu \mathrm{m}$ \\
Integral scale $L_{i}$ & $3 \mathrm{~cm}$ \\
\hline \hline
\end{tabular}



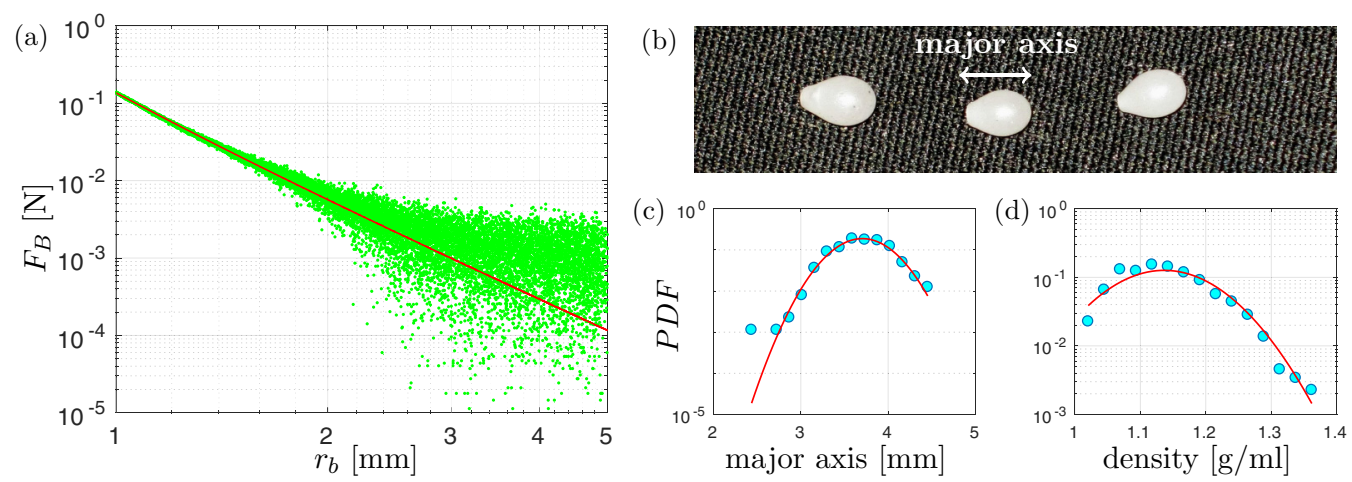

FIG. 3. (a) Magnetic force $F_{B}$ between the magnets as a function of their separation distance $r$. (b) Photograph of some beads coated with wax. (c) Main axis length probability distribution function (PDF) and (d) density PDF, with their Gaussian fits.

\section{EXPERIMENTAL PROCEDURES AND MEASUREMENTS}

In order to relate the size of the aggregates to the intensity of turbulence, different experimental runs were performed at different rotation frequencies. At the beginning of each experiment, all the magnetic beads are placed inside the tank packed in a single large cluster and then the disks are set into rotation. Under the action of turbulence, the initial large aggregate breaks into smaller ones of different sizes. Note that even if we cannot totally exclude that fragmentation takes place by collision on the grids, essentially we have visually observed it in the core of the flow. As already said, the aggregation rate for the small solid fraction of 3\% is quite low. Aggregation may arise in the bulk but takes place mainly close to the grids where the probability of aggregate collision is higher. Therefore, after some time, a stationary state [16] is reached when this aggregation process and the turbulent fragmentation in the bulk balance. For the smallest aggregates, the cohesive force is larger than the turbulent stresses preventing their fragmentation towards smaller scales. Fragmentation is thus blocked or frozen at a certain aggregate mean size where on average the cohesive force equilibrates the hydrodynamical force which is a function of the dissipation rate $\epsilon$ of turbulence. This force balance is obtained when the last bond between two parts of an aggregate breaks. For each frequency, when the stationary regime is achieved (and checked by visual inspection), we record with two synchronized CCD cameras, a 255- or 500-frame video sequence of the flow at a rate of 5 images per second. We choose on purpose a low video rate in order to perform statistics of the aggregate size from uncorrelated images. Three different regimes are detected and illustrated in Fig. 4. As expected, at a low frequency [8.5 Hz in Fig. 4(a)] the aggregates are made of a relatively large number of magnets, while at higher frequency, the size of the aggregates becomes smaller [see Fig. 4(b) for $14.5 \mathrm{~Hz}$ ] until the flow is seeded by merely only individual particles [see Fig. 4(c) for $19 \mathrm{~Hz}$.

To measure the volumes of the aggregates, we have performed a three-dimensional digital reconstruction of each of them using the convex hull volume reconstruction method [17]. As shown in Ref. [18], the precision of the volume reconstruction increases with the number of cameras. Here with only two cameras, the volumes are always overestimated. However, most of the time the shape of the aggregates is not concave; then this error should not impact our results. Moreover, the packing coefficients, introduced later in the next section, will take into account the eventual concavity of the aggregates. The principle of the reconstruction is the following: A 10-cm-side initial cubic volume is determined at the center of the tank, a region seen by both cameras. This volume is then divided in eight cubes with equal side which are projected on the two images. If the projection of a cube is empty in at least one image, this cube is discarded. Otherwise, this cube is divided into eight smaller cubes and the process is repeated until the current cube is completely filled and then saved. Finally, the volume of each aggregate is determined using the positions and the sizes of each saved cube. 


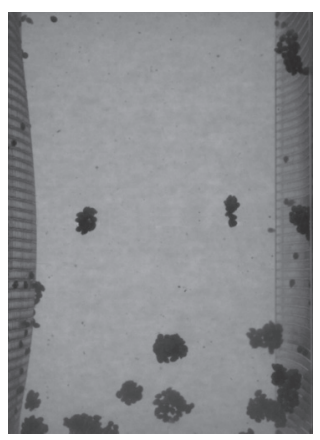

(a)

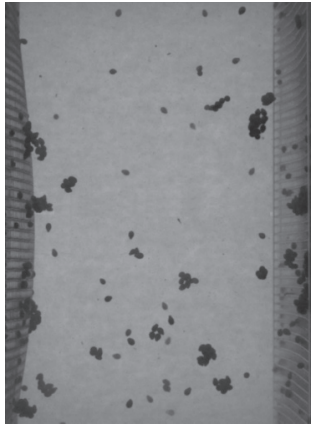

(b)

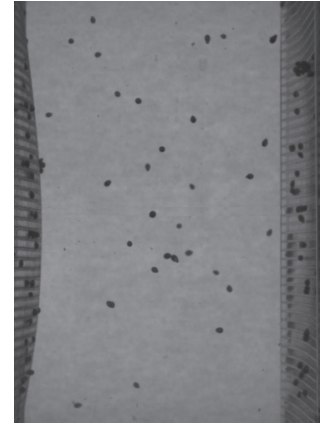

(c)

FIG. 4. Observation of the three different regimes as a function of the turbulence intensity: (a) $f=8.5 \mathrm{~Hz}$, (b) $f=14.5 \mathrm{~Hz}$, and (c) $f=19 \mathrm{~Hz}$.

\section{MODEL FOR THE AVERAGE AGGREGATE SIZE}

On average, aggregates consist of a number $N$ of magnetic particles of diameter $d$. We can then express their average volume $V$, cross section area $S$, and length $L$ as a function of $N$ and $d$,

$$
V=c_{3} N^{3 / d_{3}} d^{3}, \quad S=c_{2} N^{2 / d_{2}} d^{2}, \quad L=c_{1} N^{1 / d_{1}} d,
$$

where $1 / d_{1}, 2 / d_{2}$, and $3 / d_{3}$ are fractal dimensions characterizing the shapes of the aggregates and $c_{1}$, $c_{2}$, and $c_{3}$ three parameters representing their level of packing. From the inspection of the aggregates as can be seen in Fig. 4, they appear to be quite compact without any arborescence. As a consequence, and in order to simplify our analysis, we will assume in the following that $d_{3}=3$ and $c_{3}=\pi / 6$. In this way, the volume of an aggregate of $N$ spheres is simply equal to $N$ times the volume of a sphere. This will keep the possible fractal arrangements of clusters only in the expressions of $S$ and $L$. Following the seminal idea of Kolmogorov [19] concerning drop breakage in turbulence, we model now the mean size of the aggregates by the balance between the hydrodynamic force $F_{H}$ acting on their cross section and the magnetic force $F_{B}$ that tends to keep two particles clustered together. Indeed, when an aggregate breaks into smaller ones, the last link between the initial aggregate and a particle (belonging or not to another cluster) is controlled by a unique dipolar interaction, i.e., by the force $F_{B}$. This assumption differs strongly from the classical hypothesis where the number of broken bonds scales with the number of aggregated particles [20]. To estimate $F_{H}$, we will use the results of Qureshi et al. [21] and Volk et al. [22], who show that the variance of the fluctuations of acceleration of neutrally buoyant inertial particles is proportional to the pressure fluctuation variance at the aggregate scale (the viscous term being negligible because of the high value of the aggregate Reynolds number). Thus, here the inertial terms in the momentum equation are responsible first for the transport of the aggregates but also for their eventual breaking. Note that in the model by Kolmogorov [19], the mean drop size is obtained by replacing in the force balance the magnetic force by the capillary force. Therefore, for an aggregate of size $L$ within the inertial range of turbulence, the hydrodynamic force $F_{H}$ is given by

$$
F_{H} \sim \frac{1}{2} \rho\left(\delta u_{L}\right)^{2} S,
$$

where $\delta u_{L}$ is the typical velocity increment at scale $L$ and $\rho$ the fluid density. Taking into account that $\delta u_{L} \sim(\epsilon L)^{1 / 3}$, which is the classical law of homogeneous and isotropic turbulence [11] together with our measurement of the dissipation rate $\epsilon$, we can express the equilibrium regime of the fragmentationaggregation process

$$
F_{H} \sim 2 \rho(\alpha \pi f)^{2}\left(R^{2} d^{4} N^{\beta}\right)^{2 / 3} c_{2} c_{1}^{2 / 3}=F_{B},
$$

with $\beta=\left(3 d_{1}+d_{2}\right) / d_{1} d_{2}$. 
For high rotation rates of the impellers, we have observed that aggregates are completely fragmented and that only individual particles are present in the flow [see Fig. 4(c)]. Therefore, aggregates reach their smallest size when they consist of only $N_{c}=2$ beads, i.e., chainlike structures implying that $c_{1}=1, c_{2}=\pi / 4, c_{3}=\pi / 6, d_{1}=1$, and $d_{2}=2$ giving $\beta_{c}=5 / 2$. A threshold frequency $f_{c}$ at the onset is thus obtained from Eq. (3). For our setup, we calculate a value $f_{c} \approx 25 \mathrm{~Hz}$. This value corresponds to a dissipation rate $\epsilon_{c} \sim 1 \mathrm{~m}^{2} / \mathrm{s}^{3}$. Using this threshold, we can recast Eq. (3) to predict the average number of particles per aggregate

$$
N \sim\left(\frac{f_{c}}{f}\right)^{3 / \beta}\left(\frac{N_{c}}{a}\right)^{\beta_{c} / \beta} \text { or } N \sim\left(\frac{\epsilon_{c}}{\epsilon}\right)^{1 / \beta}\left(\frac{N_{c}}{a}\right)^{\beta_{c} / \beta},
$$

with $a=\left(4 / \pi c_{1}^{2 / 3} c_{2}\right)^{3 / 2 \beta_{c}}$. As $\beta_{c}=5 / 2, a=\left(4 / \pi c_{1}^{2 / 3} c_{2}\right)^{3 / 5}$, a value that remains close to 1 for the different arrangements that can be encountered when packing beads [23]. Note that the proportionality constant that appears through the $\sim$ symbol in Eq. (4), originates from the definition of $F_{H}$ [Eq. (2)], and can be absorbed in constant $a$.

As can be observed in Fig. 4(a), in the low-frequency or weakest-turbulence regime, the aggregates seem quite smooth and compact. In this case $d_{1}=d_{2}=3$ and $\beta=4 / 3$. As a consequence, Eq. (4) leads to a scaling law valid far from the onset for the average number $N$ of particles per aggregate as a function of the rotation frequency $f$ of the disks or of the dissipation rate of turbulence:

$$
N \propto\left(\frac{f}{f_{c}}\right)^{-9 / 4} \text { or } N \propto\left(\frac{\epsilon}{\epsilon_{c}}\right)^{-3 / 4} .
$$

A similar scaling law is predicted in [20] but the assumption about the number of bonds broken at fragmentation leads to a different exponent. These results are analogous to the pioneer work of Kolmogorov on the drop fragmentation in turbulence where the size of the droplet $D_{\text {drop }}$ is related to the turbulent intensity $D_{\text {drop }} \sim \epsilon^{-K}$ with $K=2 / 5$ [19].

\section{COMPARISON BETWEEN EXPERIMENTAL RESULTS AND THEORY}

After having processed the synchronized video images issued from the two cameras, a statistical study of the aggregate size and shape distribution is realized. Note that as our model applies only for $N \geqslant 2$, the individual particles are not taken into account in the statistical analysis. First, Fig. 5(a) presents the evolution of the measured mean number $\left\langle N_{\text {expt }}\right\rangle$ of particles per aggregate for the two different densities of fresh and salty water. The measured mean number $\left\langle N_{\text {expt }}\right\rangle$ is calculated from the volume of each aggregate divided by the volume $\pi d^{3} / 6$ of a single particle. It is an upper bound of the real number of particles $N$ that depends on the packing number $c_{3}:\left\langle N_{\text {expt }}\right\rangle=6 c_{3} / \pi N$ with $c_{3}$ between $\pi / 6$ for chains and $1 / \sqrt{2}$ for the maximum admissible packing of spheres [23]. We can conclude that the value of $c_{3}$ is quite constrained and stays in any case close to 0.5 , thus justifying our choice to keep $c_{3}=\pi / 6$. Then, from the measurement of the surface $S$ and the length $L$ of an aggregate, we can determine $d_{1}$ and $d_{2}$ and then calculate $\beta$, which is plotted in Fig. 5(a) versus $\epsilon / \epsilon_{c}$. To validate our model, we want to determine the aggregate mean size from the evolution of $\beta$. To do so, we use a smooth sigmoid function $\beta_{\text {fit }}=\beta_{0} /\left\{1+\exp \left[\left(\epsilon-\epsilon_{b}\right) / \sigma\right]\right\}+\beta_{\infty}$ that fits the experimental values of $\beta$. From this fit, we can then determine the evolution of $\left\langle N_{\text {expt }}\right\rangle$ as a function of $\epsilon / \epsilon_{c}$, with only one adjustable parameter $a$ which takes into account the packing coefficients and also some proportionality constants (close to 1) coming from the definition of $F_{H}$. We see that our theoretical model nicely reproduces the experimental results on the entire range of exploration [solid lines in Fig. 5(a)] with a single fitting coefficient $a=0.9$. Between the large and smooth aggregate asymptotic regime given by the $-3 / 4$ scaling law (5) and the threshold, there exists a smooth transition where the shape of the aggregates becomes less regular, acquiring nontrivial fractal dimensions. Finally, under the threshold, the average measured number $\left\langle N_{T}\right\rangle$ of aggregated or not particles becomes close to the single-particle limit as can be observed in Fig. 5(b). We also 
(a)

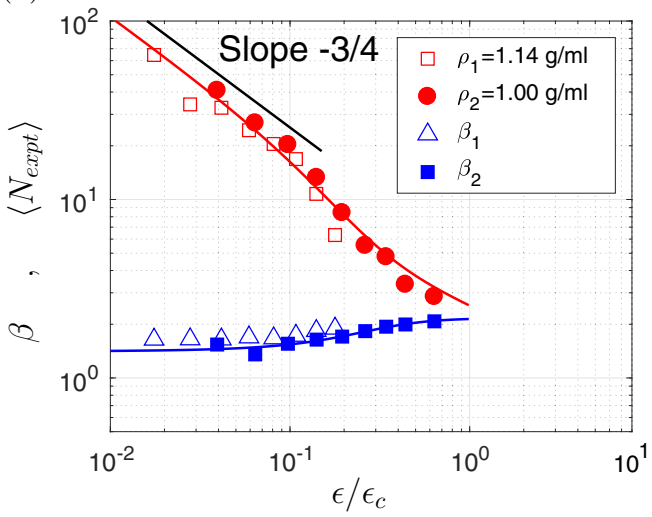

(b)

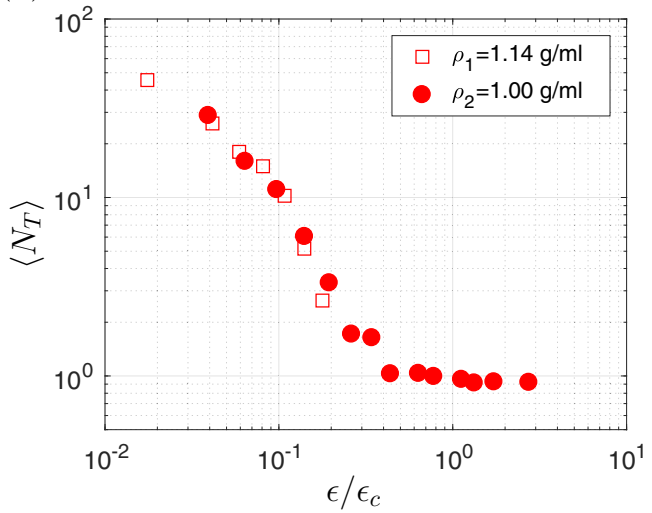

FIG. 5. (a) Experimental results for fresh water are shown by red closed circles $\left(\left\langle N_{\text {expt }}\right\rangle\right)$ and blue closed squares $(\beta)$ and the salt water results are shown by red open squares $\left(\left\langle N_{\text {expt }}\right\rangle\right)$ and blue open triangles $(\beta)$. Also shown are the theoretical predictions (solid lines) of the average number of particles $N$ per aggregate versus the dissipation rate $\epsilon$ of turbulence. A smooth sigmoid evolution for $\beta$ is fitted through the fresh water data and is used together with the measured threshold $f_{c}=25$ and fitting parameters $a=0.9$ and $6 c_{3} / \pi=1$ to predict $\left\langle N_{\text {expt }}\right\rangle$. (b) Measure of the average number $\left\langle N_{T}\right\rangle$ of particles per aggregate taking into account individual particles in the statistics.

observe that the results of the experiments using fresh and salty water are very close one to the other, justifying our assumption to neglect the settling.

Therefore, our results validate our hypothesis that aggregates break one bond after another, reaching their average final size when the last bond breaks. As explained in Ref. [24], a hierarchical fragmentation could indeed lead to a log-normal distribution of the aggregate sizes. Even if the number of aggregates that we have analyzed is limited, we have computed the probability density functions (PDFs) of the number $N_{\text {expt }}$ of particles in each aggregate for different rotation rates of the

(a)

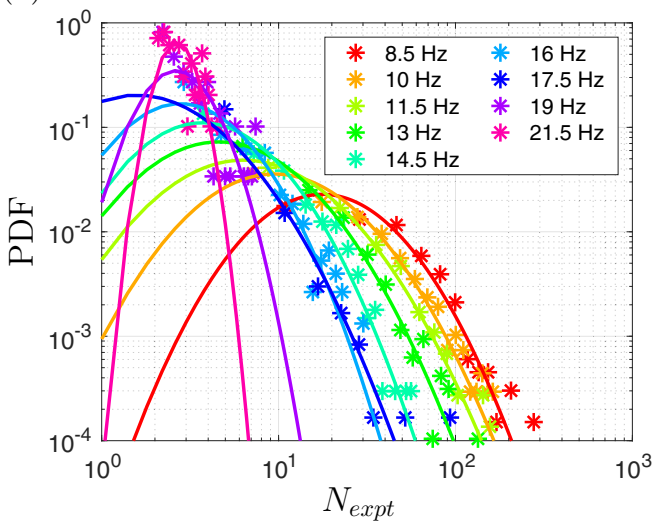

(b)

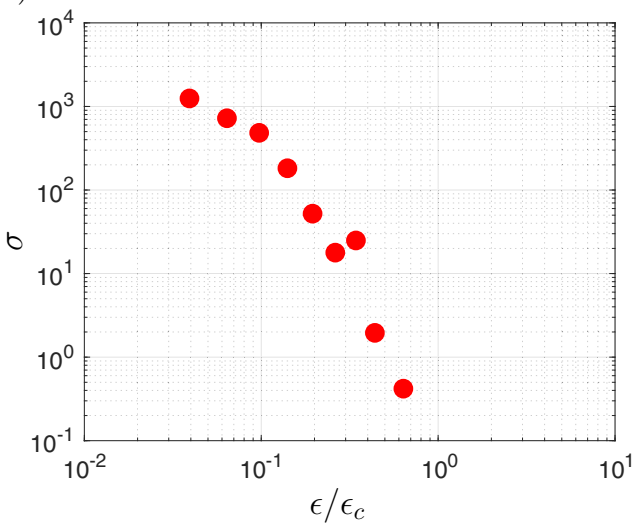

FIG. 6. (a) Probability density functions of the size of the aggregates for different rotation rates of the impellers in the case of the fresh water experiments. In agreement with a hierarchical breaking mechanism, log-normal distributions that have the same means and standard deviations as the experimental distributions have been plotted through each distribution. (b) Evolution of the variance $\sigma$ of the PDFs (fresh water) as a function of the dissipation rate $\epsilon$ of turbulence. 
impellers for the experiments performed in fresh water, discarding as before individual particles in the statistics. Figure 6(a) shows these experimental distributions. We have checked that their mean values and variances are converged, which is enough to completely determine the shape of log-normal distributions which are also plotted in Fig. 6(a) (solid lines) without any adjustable parameter. As can be seen in the figure, there is good agreement between the log-normal PDFs and the experimental data. The tails of the PDFs show a monotonic increase of the large cluster population as the intensity of turbulence decreases, a trend that can also be observed in Fig. 6(b) on the evolution of the variance $\sigma$ of the PDFs versus the dissipation rate $\epsilon$ of turbulence. However, to fully validate the log-normal distributions of the aggregate sizes, more extensive measurements would be needed in order to converge the tails of the experimental PDFs.

\section{CONCLUSION}

In classical studies on aggregation of nano- or microparticles in turbulent flows, the particles are usually smaller than Kolmogorov's scale of turbulence and thus are essentially sensitive to viscous effects. In this study, we have deliberately chosen to study the fragmentation of particle aggregates whose sizes are within the inertial range of turbulence. Experiments were performed using in-house manufactured millimetric magnetic particles that aggregate due to their magnetic field. The consideration of a classical model of turbulence based on Kolmogorov's similarity hypothesis leads to the prediction of the average size of the aggregates as a function of the turbulence intensity. Experimental measurements, using three-dimensional aggregate detection and reconstruction software, and theoretical predictions agree very well. The analysis of the aggregate size distribution is closed to a log-normal distribution and is coherent with the hierarchical physical mechanism we evoke for aggregate fragmentation. As a conclusion, we claim that knowing the intensity of turbulence and knowing the cohesive force and shapes of the particles, our model can predict the average size of the aggregates. We expect that our result can be used in different engineering situations, but we claim also that it can be used to probe turbulence itself: Knowing the force field between particles, the sole observation of the aggregate sizes and shapes should lead to the estimation of the level of turbulence.

\section{ACKNOWLEDGMENTS}

This work was carried out in the framework of the Labex MEC Project (No. ANR-10- LABX-0092) and of the A*MIDEX Project (No. ANR-11-IDEX-0001-02), funded by the "Investissements d'Avenir" French Government program managed by the French National Research Agency. H.M.D.L.R. would like to thank CONACYT for his international Ph.D. grant.

[1] F. Lundell, L. D. Söderberg, and P. H. Alfredsson, Fluid mechanics of papermaking, Annu. Rev. Fluid Mech. 43, 195 (2011).

[2] C. B. Miller, Biological Oceanography (Wiley, New York, 2009).

[3] J. Blum and G. Wurm, The growth mechanisms of macroscopic bodies in protoplanetary disks, Annu. Rev. Astron. Astrophys. 46, 21 (2008).

[4] M. U. Bäbler, M. Morbidelli, and J. Bałdyga, Modelling the breakup of solid aggregates in turbulent flows, J. Fluid Mech. 612, 261 (2008).

[5] A. Pumir and M. Wilkinson, Collisional aggregation due to turbulence, Annu. Rev. Condens. Matter Phys. 7, 141 (2016).

[6] B. Oyegbile, P. Ay, and S. Narra, Flocculation kinetics and hydrodynamic interactions in natural and engineered flow systems: A review, Env. Eng. Res. 21, 1 (2016). 
[7] F. Ravelet, Bifurcations globales hydrodynamiques et magnetohydrodynamiques dans un écoulement de von Kármán turbulent, Ph.D. thesis, Ecole Polytechnique X, 2005.

[8] N. Machicoane, R. Zimmermann, L. Fiabane, M. Bourgoin, J.-F. Pinton, and R. Volk, Large sphere motion in a nonhomogeneous turbulent flow, New J. Phys. 16, 013053 (2014).

[9] W. Thielicke and E. J. Stamhuis, PIVlab-Towards user-friendly, affordable and accurate digital particle image velocimetry in MATLAB, J. Open Res. Softw. 2, e30 (2014).

[10] S. B. Pope, Turbulent flows, Meas. Sci. Technol. 12, 2020 (2001).

[11] A. N. Kolmogorov, Dissipation of energy in locally isotropic turbulence, Dokl. Akad. Nauk SSSR 32, 19 (1941).

[12] D. Xu and J. Chen, Accurate estimate of turbulent dissipation rate using PIV data, Exp. Thermal Fluid Sci. 44, 662 (2013).

[13] K. R. Sreenivasan, On the universality of the Kolmogorov constant, Phys. Fluids 7, 2778 (1995).

[14] R. Labbé, J.-F. Pinton, and S. Fauve, Power fluctuations in turbulent swirling flows, J. Phys. (France) II 6, 1099 (1996).

[15] L. D. Landau, J. S. Bell, M. J. Kearsley, L. P. Pitaevskii, E. M. Lifshitz, and J. B. Sykes, Electrodynamics of Continuous Media (Elsevier, Amsterdam, 2013).

[16] R. D. Vigil, On equilibrium solutions of aggregation-fragmentation problems, J. Colloid Interface Sci. 336, 642 (2009).

[17] K.-m. (G.) Cheung, S. Baker, and T. Kanade, Shape-from-silhouette across time part I: Theory and algorithms, Int. J. Comput. Vision 62, 221 (2004).

[18] D. Adhikari and E. K. Longmire, Visual hull method for tomographic PIV measurement of flow around moving objects, Exp. Fluids 53, 943 (2012).

[19] A. N. Kolmogorov, On the breakage of drops in a turbulent flow, Dokl. Akad. Nauk SSSR 66, 825 (1949).

[20] D. H. Bache, Floc rupture and turbulence: A framework for analysis, Chem. Eng. Sci. 59, 2521 (2004).

[21] N. M. Qureshi, M. Bourgoin, C. Baudet, A. Cartellier, and Y. Gagne, Turbulent Transport of Material Particles: An Experimental Study of Finite Size Effects, Phys. Rev. Lett. 99, 184502 (2007).

[22] R. Volk, E. Calzavarini, E. Lévêque, and J.-F. Pinton, Dynamics of inertial particles in a von Kármán turbulent flow, J. Fluid Mech. 668, 223 (2011).

[23] J. H. Conway and N. J. A. Sloane, Sphere Packings, Lattices, and Groups, 2nd ed. (Springer, New York, 1993).

[24] E. Villermaux, Fragmentation, Annu. Rev. Fluid Mech. 39, 419 (2007). 